\title{
Intrathecal reboxetine suppresses evoked and ongoing neuropathic pain behaviours by restoring spinal noradrenergic inhibitory tone
}

\author{
Sam Hughes ${ }^{a}$, Louise Hickey ${ }^{a}$, Lucy F. Donaldson ${ }^{b}$, Bridget M. Lumb ${ }^{a, \star}$, Anthony E. Pickering ${ }^{a}$
}

\begin{abstract}
The descending noradrenergic (NAergic) projection to the spinal cord forms part of an endogenous analgesic system. After nerve injury, a localised failure in this compensatory system has been implicated as a permissive factor in the development of neuropathic sensitisation. We investigated whether restoring descending NAergic tone with intrathecal reboxetine can oppose the development of the neuropathic pain phenotype after tibial nerve transection (TNT). Rats had a lumbar intrathecal catheter implanted at the time of nerve injury for administration of reboxetine $(10 \mu \mathrm{g})$ in both acute and chronic dosing experiments. In acute dosing experiments, both intrathecal and systemic $(30 \mathrm{mg} / \mathrm{kg}$ ) reboxetine partially reversed mechanical allodynia. This antiallodynic effect of intrathecal reboxetine was blocked by prior administration of yohimbine $(\alpha 2$-adrenoceptor antagonist, $30 \mu \mathrm{g})$ but not by prazosin $(\alpha 1$-adrenoceptor antagonist, $30 \mu \mathrm{g})$ or propranolol ( $\beta$-adrenoceptor antagonist, $100 \mu \mathrm{g})$. Chronic intrathecal reboxetine $(10 \mu \mathrm{g}$, intrathecally, twice daily for 2 weeks) suppressed the development of cold and mechanical allodynia. Nerve-injured animals demonstrated a place preference for intrathecal reboxetine, suggesting that it also reduced spontaneous pain. In contrast, an equivalent antiallodynic dose of systemic reboxetine (30 mg/kg) was aversive in both naive and TNT rats. On cessation of chronic intrathecal reboxetine, there was a gradual development of allodynic sensitisation that was indistinguishable from control TNT animals by 7 days after the end of dosing. Our results suggest that pharmacological restoration of spinal NAergic tone with intrathecal reboxetine can suppress both allodynia and spontaneous pain in the TNT model.
\end{abstract}

Keywords: Neuropathic pain, Spontaneous pain, Descending control, Noradrenaline, Intrathecal noradrenergic reuptake inhibitor

\section{Introduction}

Neuropathic pain arising after a lesion of a peripheral nerve results in the development of increased sensitivity to applied stimuli and/or spontaneous ongoing pain. Treatment options for such neuropathic pain remain limited, ${ }^{15}$ but noradrenergic (NAergic) reuptake inhibitors are among the most clinically effective agents. ${ }^{4,41}$ However, their utility is often limited by troublesome side effects that follow systemic administration as a result of both on- and offtarget actions. ${ }^{41}$ Therefore, there has been considerable interest in dissecting the mechanism of action of the central NAergic system in pain control, particularly during the development of neuropathic sensitisation, with a view to identify better means of therapeutic intervention. ${ }^{32,38}$

\footnotetext{
Sponsorships or competing interests that may be relevant to content are disclosed at the end of this article.

a School of Physiology and Pharmacology, University of Bristol, Bristol, United Kingdom, ${ }^{b}$ Arthritis Research UK Pain Centre and School of Life Sciences, The Medical School QMC, University of Nottingham, Nottingham, United Kingdom

${ }^{*}$ Corresponding author. Address: School of Physiology and Pharmacology, Medical Sciences Building, University Walk, University of Bristol, BS8 1TD, United Kingdom. Tel.: 0117331 2313; fax: +44 (0)11733 11889. E-mail address: b.m.lumb@bristol. ac.uk (B. M. Lumb).

PAIN 156 (2015) 328-334

(C) 2015 International Association for the Study of Pain. This is an open access article distributed under the Creative Commons Attribution- Non Commercial License, where it is permissible to download, share and reproduce the work in any medium, provided it is properly cited. The work cannot be used commercially.

http://dx.doi.org/10.1097/01.j.pain.0000460313.73358.31
}

A population of pontine NAergic neurons with descending projections to the spinal cord is thought to regulate acute pain processing. ${ }^{22,23,26,49}$ After peripheral nerve injury, changes within this descending NAergic control system have been implicated in the development of neuropathic pain. ${ }^{19,25,39,46}$ Intriguingly, differences in the ability to engage this NAergic control system have been linked to the variable expression of neuropathic sensitisation in several nerve-injury models and across rat strains. ${ }^{9,50}$ The descending NAergic system acts to delay the appearance of neuropathic signs in the acute phase after nerve injury but then fails to prevent the onset of sensitisation because of a progressive loss of spinal NAergic tone, ${ }^{24}$ although it still acts to spatially limit the spread of sensitisation from the injured nerve territory.

Strategies to augment NAergic tone through the use of reuptake inhibitors have been partially effective in alleviating neuropathic allodynia in experimental models when administered systemically ${ }^{5,6,14}$ and also when given intrathecally (i.t.). ${ }^{36}$ This latter approach is of particular interest because many of the troublesome side effects of the reuptake inhibitors (such as sedation, dizziness, and anxiety) are thought to be mediated at supratentorial sites, which could be minimised by intrathecal dosing. However, effectiveness of chronic intrathecal administration of a selective NAergic reuptake inhibitor on the development of neuropathic pain has yet to be explored.

Given that there is evidence for a loss of descending spinal NAergic tone after nerve injury, we aimed to test the efficacy of chronic dosing with spinal reboxetine (selective reuptake inhibitor) to see whether it could prevent or attenuate the development of neuropathic sensitisation. We show that intrathecal reboxetine 
acts to effectively suppress neuropathic sensitisation through an $\alpha 2$-mediated mechanism. Furthermore, we show that intrathecal reboxetine induces a place preference in nerve-injured (but not naive) animals, suggesting that it attenuates ongoing spontaneous pain, ${ }^{27}$ whereas an equivalently effective antiallodynic dose of systemic reboxetine produces aversion in both tibial nerve transection (TNT) and naive animals.

\section{Materials and methods}

\subsection{Animals}

Experiments were performed on male Wistar rats ( $n=55$; Harlan, Bicester, United Kingdom). All procedures were licenced by the UK Home Office according to the 2010/63 Directive of European Union and adhered to the guidelines of the Committee for Research and Ethical Issues of International Association for the Study of Pain. All experimental protocols were approved by the University of Bristol local Ethical Review Panel. Animals were single housed, with an enriched environment under a standard 12-hour light-dark cycle, with ad libitum access to food and water.

\subsection{Tibial nerve transection model}

Peripheral neuropathy was induced using the TNT variant of the spared nerve-injury (SNI) model $(n=38) .{ }^{24,28}$ Under ketamine $(50 \mathrm{mg} / \mathrm{kg})$ and medetomidine $(300 \mu \mathrm{g} / \mathrm{kg})$ anaesthesia, the left hind limb was abducted and externally rotated to a lateral position, and a longitudinal incision was made at the mid-thigh level through the biceps femoris. The sciatic nerve was exposed and the sural, tibial, and common peroneal branches were carefully identified. The tibial nerve was tightly ligated with 5-0 silk and a 2-mm section was cut, taking care to avoid damage to the sural and common peroneal nerves.

\subsection{Chronic intrathecal cannulation}

A chronic intrathecal catheter was implanted at the L5-L6 interspace ${ }^{24,42,45}$ at the same time as TNT ( $n=26$, under continuous anaesthesia) and also in naive rats $(n=10)$. $A$ sterilised 32-gauge intrathecal catheter (CR3212; ReCathCo; Allison park, PA) was threaded through a 25-gauge hypodermic needle inserted between L5-L6 vertebrae until a tail flick indicated penetration of the dura. The catheter was advanced cranially 2 to $3 \mathrm{~cm}$ so the rostral tip reached the lumbar enlargement. The needle and catheter stylet were removed, and the catheter was joined to an 8-cm length of PE-10 tubing, which was sutured to the paraspinous muscle and tunnelled subcutaneously to the level of the scapulae. The catheter was externalised by attaching the PE-10 tubing to a 2-cm length of PE-50 tubing that was fixed to a back-mounted pedestal system with a screw cap (313-000BM10-SP with 6-mm side connector; Plastics One, Roanoke, VA). All intrathecal drug injections were made in a volume of $10 \mu \mathrm{L}$ using a $50 \mu \mathrm{L}$ Hamilton syringe at a rate of $\sim 0.5 \mu \mathrm{L}$ per second followed by a $17 \mu \mathrm{L}$ dead space flush with saline. Correct cannula placement was confirmed by rapid and reversible hind limb paralysis after a $20 \mu \mathrm{L}$ intrathecal lidocaine injection $(10 \mathrm{mg} / \mathrm{mL})$ at the end of the behavioural testing protocol.

\subsection{Nociceptive testing}

\subsubsection{Mechanical allodynia}

The hind paw withdrawal thresholds to tactile stimuli were assessed using calibrated von Frey filaments ranging from 0.17 to
26.0 g (TouchTest, Linton Instrumentation, Diss, United Kingdom). Briefly, rats were placed in Perspex chambers with a metal mesh floor and were allowed to habituate for 15 minutes before behavioural testing. Testing started with the $2.0 \mathrm{~g}$ von Frey filament, applied perpendicular to the plantar surface of the hind paw for 3 seconds. Withdrawal thresholds were assessed and quantified using the Dixon up-and-down method. ${ }^{7}$

\subsubsection{Cold allodynia}

Hind paw withdrawal responses to cooling stimuli were assessed using the acetone test. ${ }^{8}$ After habituation to the chamber, a $1 \mathrm{~mL}$ syringe was used to apply a drop of acetone through the mesh floor to the plantar surface of the hind paw, and a hindlimb withdrawal was scored as a positive response. Acetone testing was repeated 5 times per paw with a 2-minute interval between tests, and data are represented as percentage paw withdrawal frequency recorded in response to 5 acetone applications (PWF).

\subsection{Experimental protocols}

\subsubsection{Comparison of acute intrathecal and systemic reboxetine on mechanical allodynia}

TNT rats developed mechanical allodynia over a 10-day period. On day 10 , nerve-injured $(n=5)$ and naive $(n=5)$ rats received a single intrathecal dose of reboxetine $(10 \mu \mathrm{g})^{37}$ or saline (observer blinded), and the effects on mechanical allodynia were recorded at 5, 10, 30, 60, 180, 300, 360 minutes and 24 hours after dosing.

For comparison, in an additional group of TNT rats $(n=5)$, the effects of systemic reboxetine on mechanical allodynia were assayed at the same time points by intraperitoneal (i.p.) administration on day 10 (1 mg/kg), 12 (10 mg/kg), and 14 (30 mg/kg) each in a volume of $0.1 \mathrm{~mL} / 100 \mathrm{~g} .{ }^{29}$ In each case, the degree of sensitisation returned to its previous levels 24 hours after either intrathecal or systemic dosing.

\subsubsection{Chronic intrathecal reboxetine administration in tibial nerve transection rats}

Two groups of animals ( $n=5$ per group) had TNT surgery and implantation of chronic intrathecal catheters. Reboxetine (10 $\mu \mathrm{g}$, i.t.) or saline was given (observer blinded) at the time of nerve injury and then every day at 9 AM and 4 PM until day 15 after TNT. Mechanical and cold nociceptive testing was performed every 3 days beginning at 2 PM until day 25 after TNT.

\subsubsection{Role of spinal adrenoceptors in mediating the action of intrathecal reboxetine}

TNT rats $(n=6)$ received an intrathecal dose of a selective adrenoceptor antagonist 5 minutes before reboxetine (10 $\mu \mathrm{g}$, i.t.). The testing schedule was performed over a 6-day period with reboxetine given alone on day 8 and then with intrathecal yohimbine ${ }^{9,24,43}\left(\alpha_{2}-\mathrm{AR}\right.$ antagonist, $30 \mu \mathrm{g}$ in $10 \mu \mathrm{L} 20 \% \mathrm{DMSO}$; Tocris, United Kingdom) coadministration on day 10 , prazosin ( $\alpha_{1}$-AR antagonist, $30 \mu \mathrm{g}$ in $10 \mu \mathrm{L} 30 \%$ DMSO; Tocris ${ }^{24,43}$ ) on day 12 , propranolol ( $\beta$-AR antagonist, $100 \mu \mathrm{g}$ in $10 \mu \mathrm{L}$ saline ${ }^{11}$ ) on day 14 , and vehicle (30\% DMSO) on day 16 after TNT.

2.5.4. Place preference conditioning: intrathecal vs systemic reboxetine in tibial nerve transection and naive rats

We sought to gauge the effects of intrathecal and systemic reboxetine on "on-going" neuropathic pain using a conditioned 
place preference (CPP) paradigm ${ }^{27,44}$ between days 21 to 25 post-TNT. Comparisons were made between TNT and naive rats receiving reboxetine either i.t. (through implanted catheters) or i.p. For testing, rats had a 2 day habituation period in which they were allowed to explore all 3 chambers ( 2 conditioning chambers and a neutral connecting chamber) of the CPP environment for 15 minutes. On day 3 (preconditioning), the animals were allowed to free roam and the time spent in each chamber was assessed. On day 4 (conditioning), all rats received an injection of saline and were immediately placed in one of the pairing chambers (isolated from the other chambers) for 30 minutes (i.t.) or 45 minutes (i.p.). Four hours later, rats received an injection of reboxetine (i.t., $10 \mu \mathrm{g}$ or i.p., $30 \mathrm{mg} / \mathrm{kg}$ ) and were immediately placed in the opposite chamber for either 30 (i.t.) or 45 (i.p.) minutes. On day 5 (post-conditioning), rats were placed in the CPP box with freedom to roam between the chambers and the amount of time spent in the saline- and reboxetine-paired chambers was recorded over a 15-minute period.

\section{Statistical analysis}

The presence of sensitisation was indicated by reduction in the evoked response threshold when compared with pre-TNT or predrug baseline values, respectively, using a repeated measures 1 -way analysis of variance (ANOVA) with Dunnett multiple comparison post hoc test. Differences between treatment groups over time were determined by 2-way ANOVA with Bonferroni multiple comparison post-tests. For CPP testing, the preference for drug was calculated from the time spent in the reboxetinepaired chamber during test-preconditioning. The preference or aversion was assessed using a single sample $t$ test (against the null hypothesis of no change in preference). Statistical analysis was performed with Prism software (GraphPad Software Inc, La Jolla, CA) and levels of significance were indicated as ${ }^{\star} P<0.05$, ${ }^{\star \star} P<0.01$, and ${ }^{\star \star \star} P<0.001$, NS, not significant. Power calculations to estimate experimental group sizes based on initial pilot data were calculated using $G^{*}$ power with $\alpha=0.05$ and $\beta$ $\geq 0.8$.

\section{Results}

\subsection{Intrathecal reboxetine attenuates neuropathic sensitisation}

After TNT, rats developed mechanical hyperalgesia (Fig. 1) and cold allodynia by day 7 to 10 , which is consistent with previous reports. ${ }^{21,24}$ Single-shot intrathecal administration of reboxetine $(10 \mu \mathrm{g})$ produced a significant antiallodynic effect in nerve-injured rats tested 10 days after surgery (mechanical withdrawal threshold increased from $1.2 \pm 0.3 \mathrm{~g}$ to $5.9 \pm 1.2 \mathrm{~g}$ at 10 minutes after drug administration; $n=5, P<0.05$; Fig. 1A) lasting 60 minutes. By comparison, systemic reboxetine $(1 \mathrm{mg} / \mathrm{kg}$ or $10 \mathrm{mg} / \mathrm{kg}$, i.p.) had no significant effect on TNT sensitisation, however, a dose of $30 \mathrm{mg} / \mathrm{kg}$ produced antiallodynic effects comparable with that seen with intrathecal reboxetine (mechanical withdrawal threshold increased from $0.9 \pm 0.3 \mathrm{~g}$ to $4.7 \pm$ $0.9 \mathrm{~g}$ at 30 minutes after drug; $\mathrm{n}=5, P<0.05$; Fig. 1B).

\subsection{Chronic intrathecal reboxetine suppresses neuropathic sensitisation}

Having noted that acute intrathecal reboxetine administration produced a reduction in allodynia that was equivalent to a substantial systemic dose (Fig. 1), we tested whether repeated chronic dosing could produce a maintained suppression of sensitisation.
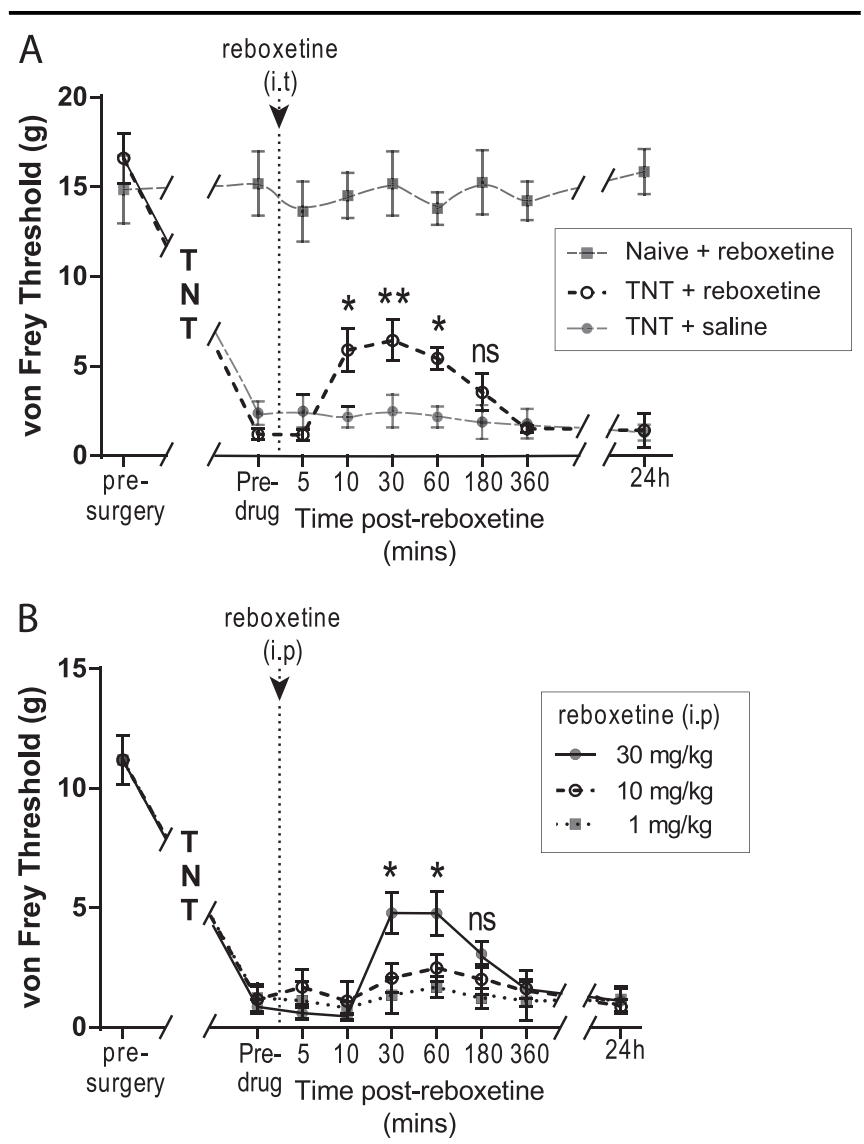

Figure 1. Intrathecal reboxetine attenuates mechanical allodynia. After tibial nerve transection (TNT), animals developed robust mechanical allodynia by day 10. (A), Intrathecal reboxetine $(10 \mu \mathrm{g})$ significantly increased mechanical paw withdrawal thresholds 10 minutes after drug administration in TNT rats but was without effect in naive rats. (B), Systemic reboxetine $(30 \mathrm{mg} / \mathrm{kg}$, i.p.) significantly increased thresholds 30 minutes after drug administration to a level similar to that seen with the intrathecal dose (lower doses were without a significant effect). Data are expressed as mean \pm SEM, $\mathrm{n}=5$ per group. Comparisons between pre- and post-reboxetine mechanical withdrawal thresholds were made using a repeated measures 1-way analysis of variance with Dunnett multiple comparison test (NS, not significant, ${ }^{\star} P<0.05,{ }^{\star \star} P<0.01$ ).

Chronic intrathecal reboxetine administered twice daily from the time of TNT prevented the development of mechanical allodynia (withdrawal threshold for the reboxetine group: $13.1 \pm 1.1 \mathrm{~g}$ vs saline: $0.8 \pm 0.5 \mathrm{~g}, \mathrm{n}=5$; $P<0.001$; Fig. $2 \mathrm{~A}$ at day 10 ) and cold allodynia (withdrawals for the reboxetine group $32.0 \% \pm 8.0 \%$ vs saline $84.0 \% \pm 7.5 \%, \mathrm{n}=5 ; P<0.001$; Fig. $2 \mathrm{~B}$ at day 10$)$. These antiallodynic actions were maintained during the whole 2-week dosing schedule.

\subsection{Reboxetine reduces neuropathic sensitisation through spinal $\alpha 2$-adrenoceptors}

The identity of the receptor mediating the antiallodynic effects of intrathecal reboxetine was investigated by spinally coadministering adrenoceptor antagonists. As previously noted, reboxetine alone significantly increased the mechanical withdrawal threshold in TNT animals (predrug: $0.5 \pm 0.1 \mathrm{~g}$ vs reboxetine: $6.3 \pm 1.5 \mathrm{~g}$; $\mathrm{n}=6, P<0.001$; Fig. 3). However, this effect was significantly attenuated by prior administration of yohimbine $(0.8 \pm 0.5 \mathrm{~g}$; $\mathrm{n}=6, P<0.001$ compared with reboxetine alone; Fig. 3), but not by prazosin (30 $\mu \mathrm{g})$, propranolol $(100 \mu \mathrm{g})$, or vehicle (30\% DMSO) administered to the same animals, indicating involvement of spinal $\alpha 2$-adrenoceptors in the action of reboxetine. 

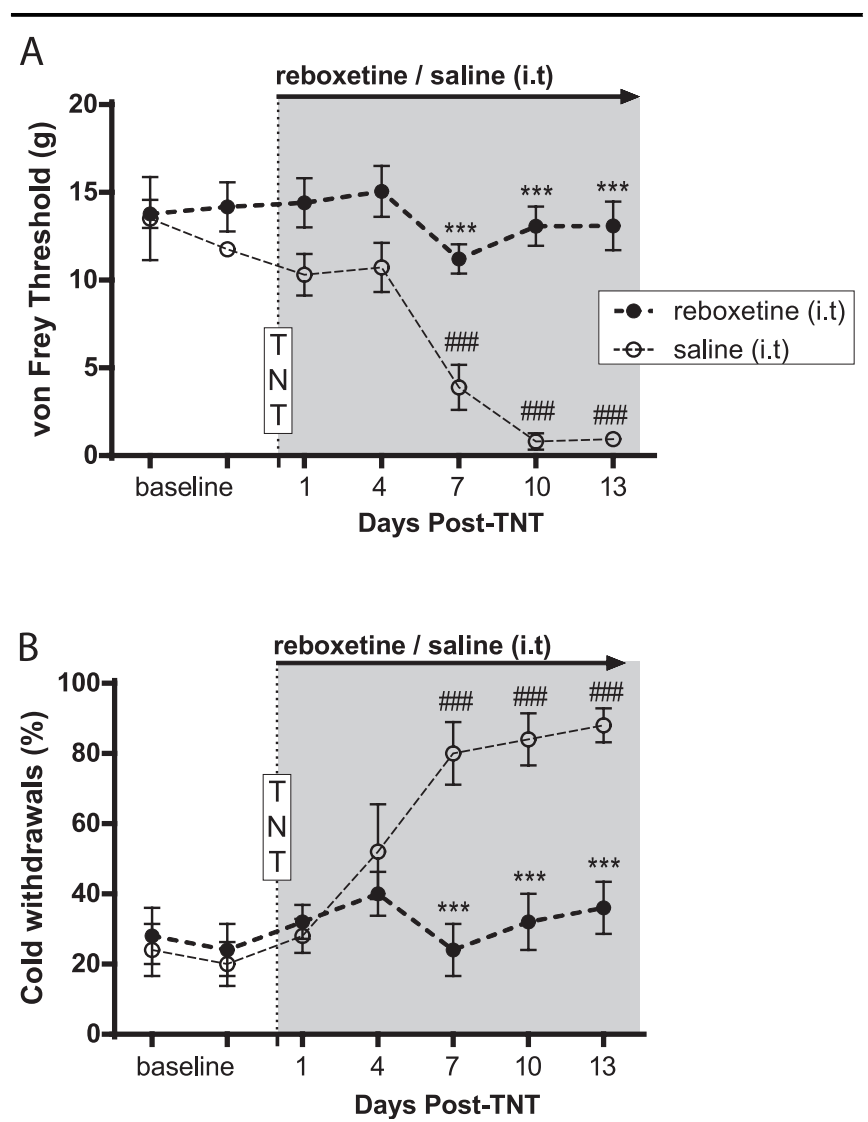

Figure 2. Prophylactic intrathecal reboxetine suppresses the development of neuropathic sensitisation. Tibial nerve transection rats received intrathecal reboxetine $(10 \mu \mathrm{g})$ or vehicle (saline) at the time of injury and then twice daily (9 AM and 4 PM) until day 15 after injury. In vehicle treated rats, mechanical (A) and cold (B) allodynia developed by day 7 which was not seen in the chronic reboxetine group. Data are expressed as mean \pm SEM, $n=5$ per group. Comparisons between reboxetine- and saline-treated rats using 2-way analysis of variance (ANOVA) with the Bonferroni post-test $\left({ }^{\star \star \star} P<0.001\right)$. The presence of allodynia in either the reboxetine- or saline-treated groups was determined by comparisons with pre-tibial nerve transection baseline thresholds or withdrawals using a repeated measures 1-way ANOVA with the Dunnett multiple comparison test (NS, not significant, \#\#\#P < 0.001).

\subsection{Tibial nerve transection rats exhibit a preference for intrathecal reboxetine}

We used a place preference assay to test whether intrathecal reboxetine might be acting to suppress ongoing pain in TNT animals. ${ }^{27}$ A single dose of intrathecal reboxetine $(10 \mu \mathrm{g})$ induced place preference in TNT rats with an increase in the time spent in the reboxetine-paired chamber after conditioning (117 \pm 38 seconds, $P<0.05$; Fig. 4). Importantly, reboxetine did not induce place preference in naive rats $(29 \pm 26$ seconds, NS; Fig. 4). These data indicate that intrathecal reboxetine alone is not sufficient to induce place preference in the absence of nerve injury consistent with the proposition that it is suppressing an ongoing neuropathic pain state in the TNT rats.

\subsection{Systemic reboxetine seems to be aversive in both tibial nerve transection and naive rats}

To compare the effect of systemic reboxetine, we used a similar CPP protocol with a dose that produced an equivalent degree of attenuation of evoked withdrawals (30 mg/kg, i.p., Fig. 1B) as the intrathecal dose $(10 \mu \mathrm{g})$. Systemic dosing produced the opposite

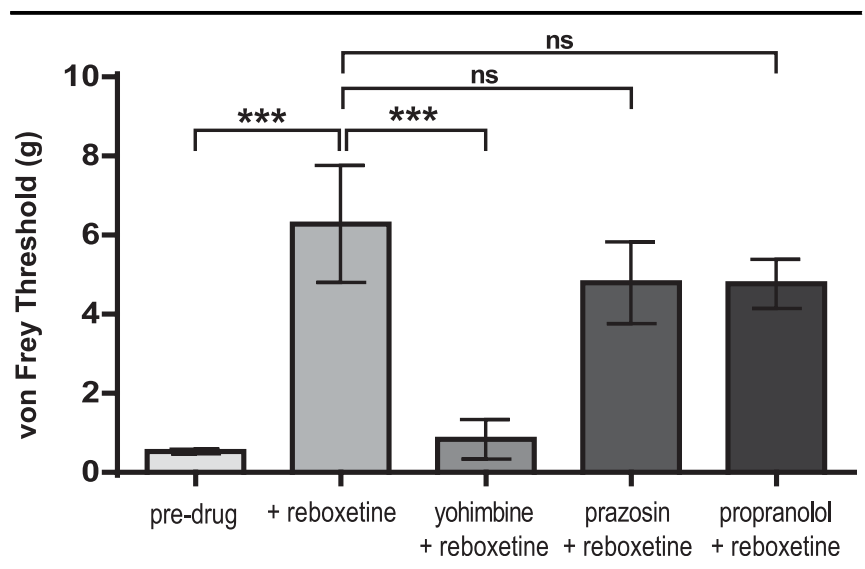

Figure 3. Intrathecal reboxetine acts through an $\alpha 2-A R$ mediated mechanism. Intrathecal reboxetine $(10 \mu \mathrm{g})$ significantly increased mechanical withdrawal thresholds in tibial nerve transection (TNT) rats. However, prior administration of yohimbine $(\alpha 2-A R$ antagonist, $30 \mu \mathrm{g})$ significantly attenuated this response. Prior administration of prazosin ( $\alpha 1$-AR antagonist, $30 \mu \mathrm{g})$, propranolol ( $\beta$-AR antagonist, $100 \mu \mathrm{g})$, and vehicle $(30 \%$ DMSO) were without an effect. Data are expressed as mean \pm SEM, $n=6$ per group. The mechanical sensitivity of TNT rats before and after reboxetine administration and in combination with intrathecal antagonists was compared using 2-way analysis of variance with the Bonferroni post-test (NS, not significant, ${ }^{* \star *} P<0.001$ ).

effect in place preference testing to that seen with intrathecal administration. In TNT rats, there was a reduction in the amount of time spent in the reboxetine-paired chamber $(-72 \pm 29$ seconds, $P<0.05$; Fig. 4). This aversive effect of systemic reboxetine was also seen in naive rats with a reduction in time spent in the reboxetine-paired chamber during the test day compared with the preconditioning day ( $-99 \pm 24$ seconds; $P<0.01$; Fig. 4).

\subsection{Neuropathic sensitisation develops after reboxetine discontinuation}

On discontinuation of the intrathecal reboxetine (after 2 weeks of twice daily dosing), both mechanical and cold sensitisation gradually developed in the ipsilateral limb over the next 7 days (Fig. 5). The mechanical allodynia was apparent from 5 days after cessation and was indistinguishable from that seen in the control group by day 8 after reboxetine withdrawal.

\section{Discussion}

We have investigated the effect of augmenting pontospinal NAergic tone, using intrathecal dosing of the selective noradrenaline reuptake inhibitor-reboxetine, ${ }^{16,37}$ on the development of the neuropathic pain phenotype after nerve injury. Using a combination of acute and chronic dosing through implanted catheters, we have shown that intrathecal reboxetine can alleviate spontaneous and evoked pain behaviours through a spinal $\alpha 2-A R$ mediated mechanism. These findings support the idea that after tibial nerve injury, there is a functional deficit in pontospinal NAergic tone, which can be reversed by chronic spinal noradrenaline (NA) reuptake inhibition.

The variable expression of a neuropathic pain phenotype across individual animals after apparently identical nerve injury is thought to be dependent on the degree of engagement of the pontospinal NAergic system. ${ }^{50}$ Differences between rat strains in the function of this NAergic system has also been suggested to play an important role in determining whether allodynia develops after nerve injury. ${ }^{9}$ A progressive functional and anatomical deficit 


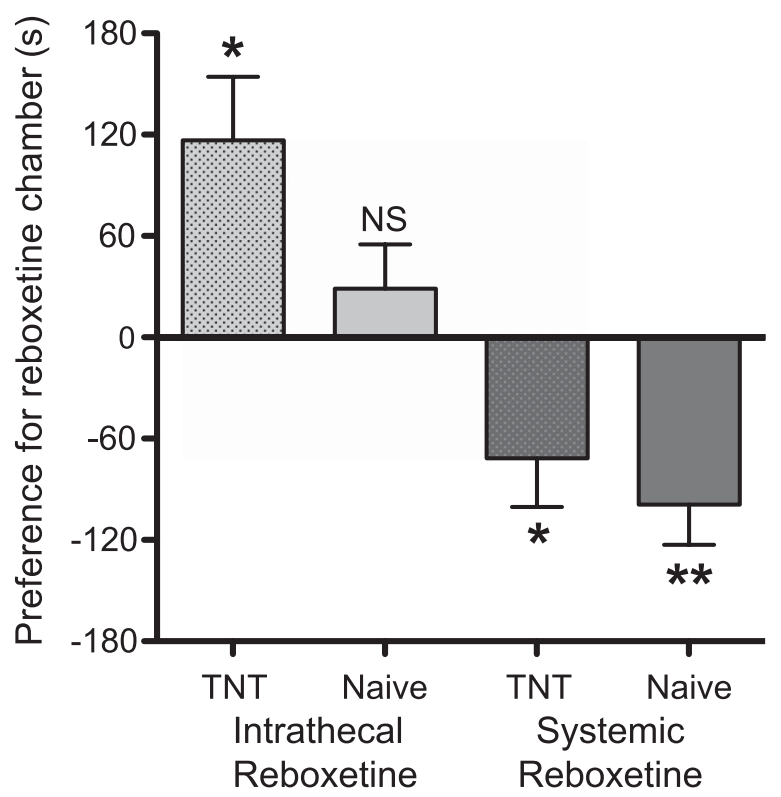

Figure 4. Nerve-injured animals exhibit a preference for intrathecal but not systemic reboxetine. In a conditioned place preference testing paradigm, the tibial nerve transection (TNT) rats (unlike naive control rats) showed a significant increase in the amount of time spent in the intrathecal reboxetine $(10 \mu \mathrm{g})$ paired chamber after conditioning. However, both TNT and naive rats showed a significant aversion to systemic reboxetine $(30 \mathrm{mg} / \mathrm{kg}$, i.p.) in the CPP paradigm with a significant reduction in the amount of time spent in the reboxetine-paired chamber after conditioning. Preference data shown as time spent in reboxetine-paired chamber on test day_-preconditioning day. Data expressed as mean \pm SEM, $n=7$ per group (NS, not significant, ${ }^{\star} P<0.05$, ${ }^{\star \star} P<0.01$; single sample, 2-tailed $t$ tests).

in the descending NAergic system has been found to accompany the development of allodynia after nerve injury. ${ }^{24}$ Inhibition of the descending NAergic system once neuropathic pain has developed is either ineffective (perhaps because of a floor effect) ${ }^{22,25}$ or amplifies the sensitisation. ${ }^{19,24}$ Therefore, we addressed the question of whether selective augmentation of spinal NAergic control could be a useful therapeutic strategy.

Acute intrathecal administration of reboxetine alleviates evoked hypersensitivity (to an equivalent degree as a substantial systemic dose), which is in line with previous reports for acute intrathecal dosing of the conus peptide Xen2174 (selective NET inhibitor) in the chronic constriction injury model. ${ }^{36}$ The question of which spinal adrenoceptors are involved in the antiallodynic action of systemic monoamine reuptake inhibitors has previously been addressed with reports supporting a role for $\alpha_{2}-\mathrm{AR}^{35}$ and $\beta_{2}-A R{ }^{52}$ Here, we found that administration of an $\alpha_{2}-A R$ antagonist given i.t. alongside reboxetine suppressed the mechanical antiallodynic effect (see also Refs. 36,37) and found no evidence for a role of either $\alpha 1$ - or $\beta$-adrenoceptors. This $\alpha_{2}$-AR antagonist reversal of reboxetine actions was also seen for cold allodynia in preliminary experiments (data not shown). Furthermore, the effect of intrathecal $\alpha_{2}$-AR antagonists to block the effect of reboxetine suggests that it is acting to increase spinal levels of noradrenaline to exert its therapeutic benefit as has been demonstrated by spinal microdialysis for systemic administration of both selective (maprotiline) and nonselective noradrenaline reuptake inhibitors in a nerve-injury model. ${ }^{35}$

Clinically, patients commonly describe the presence of spontaneous pain after nerve injury ${ }^{3}$; however most preclinical assays
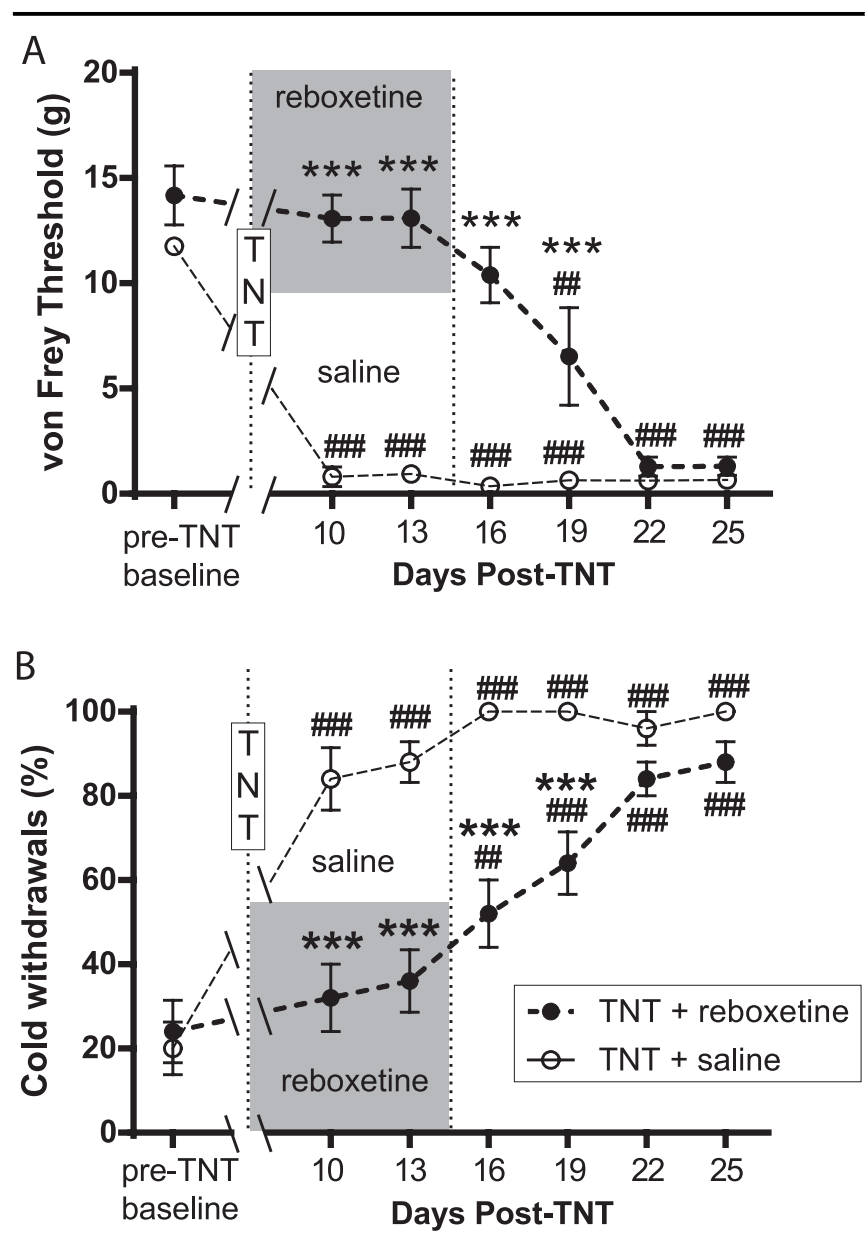

Figure 5. Chronic intrathecal reboxetine does not prevent the development of neuropathic sensitisation after discontinuation. After 2 weeks of twice daily intrathecal reboxetine $(10 \mu \mathrm{g})$ after tibial nerve transection (TNT), cessation of reboxetine dosing caused sensitisation to return over the following week, with significant mechanical sensitisation evident after 5 days (ie, by day 19). Data are expressed as mean $\pm \mathrm{SEM}, \mathrm{n}=5$ per group. Comparisons between reboxetine- and control saline-treated TNT rats over time were made using 2 -way analysis of variance (ANOVA) with the Bonferroni post-test $\left({ }^{\star \star \star} P<\right.$ 0.001). The presence of allodynia in either the reboxetine- or saline-treated groups was determined over time by comparisons with pre-TNT baseline thresholds or withdrawals using a repeated measures 1-way ANOVA with the Dunnett multiple comparison test (\#\#P<0.01, \#\#\#P<0.001).

have focussed on evoked pain measures. ${ }^{33}$ Although the occurrence of ongoing pain has been suggested to originate from spontaneous activity in peripheral nociceptors, ${ }^{10}$ there is evidence that descending facilitatory pathways play a role in the long-term maintenance of spontaneous pain. ${ }^{47}$ Here, we have used CPP testing, which has been extensively used to investigate the rewarding or aversive properties of drugs ${ }^{44}$ to investigate whether intrathecal reboxetine attenuates spontaneous pain in the TNT model using a methodology similar to that of King et al. ${ }^{27}$ Acute intrathecal reboxetine administration induced CPP in TNT rats but importantly not in naive animals, indicating that reboxetine is not rewarding in of itself when administered spinally. Therefore, this likely indicates the presence of a tonic ongoing level of pain after tibial nerve injury that can be alleviated by restoring pontospinal NAergic inhibition-consistent with the proposition that loss of descending NAergic control plays a role in the maintenance of spontaneous neuropathic pain. This is in line with a recent finding that intrathecal $\alpha 2-A R$ agonism with clonidine can also produce place preference in nerve-injured animals. ${ }^{48}$ Our findings suggest that a deficit in descending NAergic tone plays an important role in 
the expression of the neuropathic phenotype in terms of both tonic ongoing and evoked neuropathic pain, and there is consequently an imbalance between the descending inhibition and facilitation that permits the behavioural expression of sensitisation.

In the same assay, systemic reboxetine was aversive (at a dose that produced an equivalent degree of alleviation of mechanical allodynia) in both TNT and naive rats. This may be because systemic dosing of the reuptake inhibitor acts to enhance both pro- and antinociceptive actions of the central NAergic system (see Ref. 20) and therefore is relatively less effective against ongoing pain than the intrathecal route. Alternatively, this aversive action may indicate that the systemic side effects of this dose outweigh any beneficial antiallodynic action. We also noted during this testing that both of the higher doses of systemic reboxetine seemed to be associated with behaviour change suggestive of increased stress with more frequent urination and defecation. These findings chime with clinical practice where systemic dosing of NAergic reuptake inhibitors can be modestly effective but is often compromised by side effects that result in noncompliance with treatment. ${ }^{41}$

Given that the onset of ipsilateral sensitisation/allodynia after tibial nerve injury coincides with a gradual reduction in descending NAergic tone, ${ }^{24}$ we investigated the effect of chronically dosing reboxetine on the development of allodynia. The use of chronic systemic monoamine reuptake inhibitors has been shown to be more effective than acute in alleviating sensitisation after nerve injury, ${ }^{10,13,31}$ and experience with tricyclic antidepressants (TCAs) indicates that a period of 4 to 6 weeks' dosing is usually necessary before beneficial effects are seen. ${ }^{41}$ In this study, we found that chronic intrathecal dosing of reboxetine given at the time of nerve injury and then twice daily until day 15 after injury completely suppressed the development of sensitisation after tibial nerve injury. The magnitude of the beneficial effect was substantially greater than that seen with single-shot dosing of reboxetine, suggesting a summating therapeutic action (comparable to the effect of a maximal intrathecal dose of the conopeptide Xen2174 ${ }^{36}$ ). Further investigation will be required to determine whether this simply represents accumulation of the drug in the intrathecal compartment (analogous to that reported for systemic dosing ${ }^{2}$ ) or alternatively represents a form of NAergic (or downstream) neuroplasticity in sensory circuits as has been suggested from chronic systemic dosing studies with TCAs, ${ }^{1}$ perhaps involving nerve growth factors such as BDNF. ${ }^{18}$

However, once reboxetine dosing ceased, allodynic sensitisation returned within a week to the same level as that seen in control animals, suggesting that chronic reboxetine masks but does not prevent the development of a neuropathic phenotype. This slow development of sensitisation takes longer than would be expected given the known pharmacokinetics of reboxetine - again suggesting that there may be some longer-lasting plastic changes in the sensory pathways. However, the 2-week time course of dosing fails to prevent the subsequent induction of sensitisation possibly because of underlying pathological processes in other peripheral or central nociceptive pathways. In particular, the intrathecal reuptake inhibition approach would not be expected to influence peripherally generated afferent activity from nociceptors, ${ }^{10}$ which has been shown to be present for several weeks after nerve injury and may play a role in re-establishing central sensitisation. ${ }^{34,40}$ Similar findings have been reported with systemic dosing of TCAs where mechanical allodynia persisted after the cessation of dosing although other measures of evoked sensitisation were attenuated. ${ }^{1}$ It is conceivable that continuation of intrathecal dosing beyond the period of altered peripheral afferent input could prevent the subsequent development of sensitisation although this may also require that aberrant afferent barrages from the periphery have ceased in the meanwhile. ${ }^{34,40}$

The use of systemic monoamine reuptake inhibitors is established as a therapeutic strategy for chronic pain in general and neuropathic pain in particular. ${ }^{4,41}$ Animal studies have shown that such systemic dosing increases NA levels in the spinal cord. ${ }^{35}$ This raises the possibility that chronic intrathecal dosing of a reuptake inhibitor could have therapeutic advantages with an improved side effect profile. This must be set against the substantial cost and logistical challenges of chronic intrathecal delivery of drug in humans. This strategy has been implemented for clonidine infusions for neuropathic and cancer pain ${ }^{17}$ albeit complicated by postural hypotension, sedation, and bradycardia. Such side effects might be minimised by substitution of a reuptake inhibitor to augment the profile of physiologically released noradrenaline at a spinal level. Few of the selective reuptake inhibitors have had any spinal toxicity testing, ${ }^{12}$ and it is relevant to note that toxicity concerns have been raised after intrathecal administration of amitriptyline-possibly because of its off-target actions at the NMDA receptor. ${ }^{51}$ However, the Xen2174 conus compound has been reported as being safe for intrathecal administration in humans. ${ }^{30}$ There may be a role for such strategies in the treatment of severe neuropathic pain.

In summary, this study has shown a beneficial effect of spinal NA-uptake inhibition on the development of the neuropathic phenotype after peripheral nerve injury. Intrathecal reboxetine alleviated both evoked and ongoing neuropathic pain, whereas systemic administration caused an aversive response in both naive and nerve-injured rats, indicating that selective spinal administration may confer therapeutic benefits by restoring the balance of descending pain modulation.

\section{Conflict of interest statement}

The authors have no conflicts of interest to declare.

Supported by the Medical Research Council and the Wellcome Trust. A. E. Pickering is a Wellcome Trust Senior Clinical Research Fellow.

\section{Article history:}

Received 17 April 2014

Received in revised form 10 October 2014

Accepted 21 November 2014

\section{References}

[1] Arsenault A, Sawynok J. Perisurgical amitriptyline produces a preventive effect on afferent hypersensitivity following spared nerve injury. PAIN 2009;146:308-14.

[2] Assie MB, Charveron M, Palmier C, Puozzo C, Moret C, Briley M. Effects of prolonged administration of milnacipran, a new antidepressant, on receptors and monoamine uptake in the brain of the rat. Neuropharmacology 1992;31: 149-55.

[3] Backonja MM, Stacey B. Neuropathic pain symptoms relative to overall pain rating. J Pain 2004;5:491-7.

[4] Bannister K, Bee LA, Dickenson AH. Preclinical and early clinical investigations related to monoaminergic pain modulation. Neurotherapeutics 2009;6: 703-12.

[5] Berrocoso E, Mico JA, Vitton O, Ladure P, Newman-Tancredi A, Depoortere R, Bardin L. Evaluation of milnacipran, in comparison with amitriptyline, on cold and mechanical allodynia in a rat model of neuropathic pain. Eur J Pharmacol 2011;655:46-51.

[6] Bomholt SF, Mikkelsen JD, Blackburn-Munro G. Antinociceptive effects of the antidepressants amitriptyline, duloxetine, mirtazapine and 
citalopram in animal models of acute, persistent and neuropathic pain. Neuropharmacology 2005;48:252-63.

[7] Chaplan SR, Bach FW, Pogrel JW, Chung JM, Yaksh TL. Quantitative assessment of tactile allodynia in the rat paw. J Neurosci Methods 1994;53: 55-63.

[8] Choi Y, Yoon YW, Na HS, Kim SH, Chung JM. Behavioral signs of ongoing pain and cold allodynia in a rat model of neuropathic pain. PAIN 1994;59:369-76.

[9] De Felice M, Sanoja R, Wang R, Vera-Portocarrero L, Oyarzo J, King T, Ossipov MH, Vanderah TW, Lai J, Dussor GO, Fields HL, Price TJ, Porreca F. Engagement of descending inhibition from the rostral ventromedial medulla protects against chronic neuropathic pain. PAIN 2011;152:2701-9.

[10] Djouhri L, Koutsikou S, Fang X, McMullan S, Lawson SN. Spontaneous pain, both neuropathic and inflammatory, is related to frequency of spontaneous firing in intact C-fiber nociceptors. J Neurosci 2006;26: 1281-92.

[11] Durant PA, Lucas PC, Yaksh TL. Micturition in the unanesthetized rat: spinal vs. peripheral pharmacology of the adrenergic system. J Pharmacol Exp Ther 1988;245:426-35.

[12] Eisenach JC, Yaksh TL. Safety in numbers: how do we study toxicity of spinal analgesics? Anesthesiology 2002;97:1047-9.

[13] Esser MJ, Chase T, Allen GV, Sawynok J. Chronic administration of amitriptyline and caffeine in a rat model of neuropathic pain: multiple interactions. Eur J Pharmacol 2001;430:211-8.

[14] Esser MJ, Sawynok J. Acute amitriptyline in a rat model of neuropathic pain: differential symptom and route effects. PAIN 1999;80:643-53.

[15] Finnerup NB, Sindrup SH, Jensen TS. The evidence for pharmacological treatment of neuropathic pain. PAIN 2010;150:573-81.

[16] Hajos M, Fleishaker JC, Filipiak-Reisner JK, Brown MT, Wong EH. The selective norepinephrine reuptake inhibitor antidepressant reboxetine: pharmacological and clinical profile. CNS Drug Rev 2004;10:23-44.

[17] Hassenbusch SJ, Gunes S, Wachsman S, Willis KD. Intrathecal clonidine in the treatment of intractable pain: a phase I/II study. Pain Med 2002;3: 85-91.

[18] Hayashida K, Clayton BA, Johnson JE, Eisenach JC. Brain derived nerve growth factor induces spinal noradrenergic fiber sprouting and enhances clonidine analgesia following nerve injury in rats. PAIN 2008;136:348-55.

[19] Hayashida K, Peters CM, Gutierrez S, Eisenach JC. Depletion of endogenous noradrenaline does not prevent spinal cord plasticity following peripheral nerve injury. J Pain 2012;13:49-57.

[20] Hickey L, Li Y, Fyson SJ, Watson TC, Perrins R, Hewinson J, Teschemacher AG, Furue H, Lumb BM, Pickering AE. Optoactivation of locus ceruleus neurons evokes bidirectional changes in thermal nociception in rats. J Neurosci 2014;34:4148-60.

[21] Hofmann HA, De Vry J, Siegling A, Spreyer P, Denzer D. Pharmacological sensitivity and gene expression analysis of the tibial nerve injury model of neuropathic pain. Eur J Pharmacol 2003;470:17-25.

[22] Howorth PW, Teschemacher AG, Pickering AE. Retrograde adenoviral vector targeting of nociresponsive pontospinal noradrenergic neurons in the rat in vivo. J Comp Neurol 2009;512:141-57.

[23] Howorth PW, Thornton SR, O'Brien V, Smith WD, Nikiforova N, Teschemacher AG, Pickering AE. Retrograde viral vector-mediated inhibition of pontospinal noradrenergic neurons causes hyperalgesia in rats. J Neurosci 2009;29:12855-64.

[24] Hughes SW, Hickey L, Hulse RP, Lumb BM, Pickering AE. Endogenous analgesic action of the pontospinal noradrenergic system spatially restricts and temporally delays the progression of neuropathic pain following tibial nerve injury. PAIN 2013;154:1680-90.

[25] Jasmin L, Boudah A, Ohara PT. Long-term effects of decreased noradrenergic central nervous system innervation on pain behavior and opioid antinociception. J Comp Neurol 2003;460:38-55.

[26] Jones SL, Gebhart GF. Characterization of coeruleospinal inhibition of the nociceptive tail-flick reflex in the rat: mediation by spinal alpha 2-adrenoceptors. Brain Res 1986;364:315-30.

[27] King T, Vera-Portocarrero L, Gutierrez T, Vanderah TW, Dussor G, Lai J, Fields HL, Porreca F. Unmasking the tonic-aversive state in neuropathic pain. Nat Neurosci 2009;12:1364-6.

[28] Lee BH, Won R, Baik EJ, Lee $\mathrm{SH}$, Moon $\mathrm{CH}$. An animal model of neuropathic pain employing injury to the sciatic nerve branches. Neuroreport 2000;11:657-61.

[29] Leventhal L, Smith V, Hornby G, Andree TH, Brandt MR, Rogers KE. Differential and synergistic effects of selective norepinephrine and serotonin reuptake inhibitors in rodent models of pain. J Pharmacol Exp Ther 2007;320:1178-85.

[30] Lewis RJ. Discovery and development of the chi-conopeptide class of analgesic peptides. Toxicon 2012;59:524-8.

[31] McCarson KE, Ralya A, Reisman SA, Enna SJ. Amitriptyline prevents thermal hyperalgesia and modifications in rat spinal cord $\operatorname{GABA}(B)$ receptor expression and function in an animal model of neuropathic pain. Biochem Pharmacol 2005;71:196-202.

[32] Millan MJ. Descending control of pain. Prog Neurobiol 2002;66:355-474.

[33] Mogil JS. Animal models of pain: progress and challenges. Nat Rev Neurosci 2009;10:283-94.

[34] Nakagawa K, Takeda M, Tsuboi Y, Kondo M, Kitagawa J, Matsumoto S, Kobayashi A, Sessle BJ, Shinoda M, Iwata K. Alteration of primary afferent activity following inferior alveolar nerve transection in rats. Mol pain 2010;6:9.

[35] Nakajima K, Obata H, Iriuchijima N, Saito S. An increase in spinal cord noradrenaline is a major contributor to the antihyperalgesic effect of antidepressants after peripheral nerve injury in the rat. PAIN 2012;153: 990-7.

[36] Nielsen CK, Lewis RJ, Alewood D, Drinkwater R, Palant E, Patterson M, Yaksh TL, McCumber D, Smith MT. Anti-allodynic efficacy of the chiconopeptide, Xen2174, in rats with neuropathic pain. PAIN 2005;118: 112-24.

[37] Obata H, Conklin D, Eisenach JC. Spinal noradrenaline transporter inhibition by reboxetine and Xen2174 reduces tactile hypersensitivity after surgery in rats. PAIN 2005;113:271-6.

[38] Pertovaara A. Noradrenergic pain modulation. Prog Neurobiol 2006;80: 53-83.

[39] Rahman W, D'Mello R, Dickenson AH. Peripheral nerve injury-induced changes in spinal alpha(2)-adrenoceptor-mediated modulation of mechanically evoked dorsal horn neuronal responses. J Pain 2008;9: 350-9.

[40] Serra J. Microneurography: towards a biomarker of spontaneous pain. PAIN 2012;153:1989-90.

[41] Sindrup SH, Otto M, Finnerup NB, Jensen TS. Antidepressants in the treatment of neuropathic pain. Basic Clin Pharmacol Toxicol 2005;96: 399-409.

[42] Storkson RV, Kjorsvik A, Tjolsen A, Hole K. Lumbar catheterization of the spinal subarachnoid space in the rat. J Neurosci Methods 1996;65: 167-72.

[43] Takano Y, Yaksh TL. Characterization of the pharmacology of intrathecally administered alpha-2 agonists and antagonists in rats. J Pharmacol Exp Ther 1992;261:764-72.

[44] Tzschentke TM. Measuring reward with the conditioned place preference paradigm: a comprehensive review of drug effects, recent progress and new issues. Prog Neurobiol 1998;56:613-72.

[45] Veitenheimer B, Osborn JW. Role of spinal V1a receptors in regulation of arterial pressure during acute and chronic osmotic stress. Am J Physiol Regul Integr Comp Physiol 2011;300:R460-9.

[46] Viisanen $\mathrm{H}$, Pertovaara A. Influence of peripheral nerve injury on response properties of locus coeruleus neurons and coeruleospinal antinociception in the rat. Neuroscience 2007;146:1785-94.

[47] Wang R, King T, De Felice M, Guo W, Ossipov MH, Porreca F. Descending facilitation maintains long-term spontaneous neuropathic pain. J Pain 2013;14:845-53.

[48] Wei $H$, Viisanen $H$, Amorim D, Koivisto A, Pertovaara A. Dissociated modulation of conditioned place-preference and mechanical hypersensitivity by a TRPA1 channel antagonist in peripheral neuropathy. Pharmacol Biochem Behav 2013;104:90-6.

[49] West WL, Yeomans DC, Proudfit HK. The function of noradrenergic neurons in mediating antinociception induced by electrical stimulation of the locus coeruleus in two different sources of Sprague-Dawley rats. Brain Res 1993;626:127-35.

[50] Xu M, Kontinen VK, Kalso E. Endogenous noradrenergic tone controls symptoms of allodynia in the spinal nerve ligation model of neuropathic pain. Eur J Pharmacol 1999;366:41-5.

[51] Yaksh TL, Tozier N, Horais KA, Malkmus S, Rathbun M, Lafranco L, Eisenach J. Toxicology profile of $\mathrm{N}$-methyl-D-aspartate antagonists delivered by intrathecal infusion in the canine model. Anesthesiology 2008;108:938-49.

[52] Yalcin I, Tessier LH, Petit-Demouliere N, Doridot S, Hein L, Freund-Mercier MJ, Barrot M. Beta2-adrenoceptors are essential for desipramine, venlafaxine or reboxetine action in neuropathic pain. Neurobiol Dis 2009;33:386-94. 